\title{
Eficácia dos colírios ciclosporina e tacrolimo no tratamento de ceratoconjuntivite seca em cães
}

\author{
[Efficacy of cyclosporine and tacrolimus drops in the treatment of keratoconjunctivitis \\ sicca in dogs] \\ J.N. Voitena ${ }^{1}$, O. Cunha ${ }^{2}$, F.B ${ }^{2}$. Fukushima ${ }^{2}$, G.F. Carvalho ${ }^{1}$, L.C.S. Ramos ${ }^{3}$, \\ V.C. Henriques ${ }^{3}$, D.M.B. Costa ${ }^{3}$

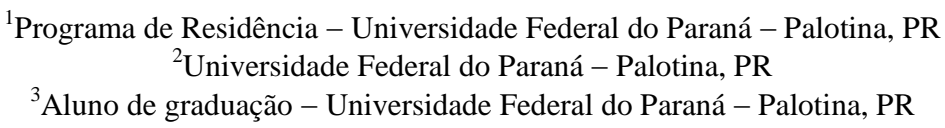

\section{RESUMO}

O objetivo deste estudo foi avaliar os efeitos do tacrolimo e da ciclosporina na produção lacrimal de cães com ceratoconjuntivite seca (CCS) durante 90 dias. Para tanto, foram utilizados colírios de tacrolimo $0,02 \%$ (TcL) e ciclosporina $0,1 \%$ (CsA) em 14 cães com CCS. Os animais foram distribuídos em dois grupos e avaliados antes do início do tratamento (T0) e aos 15 (T1), 30 (T2), 45 (T3), 60 (T4), 75 (T5) e 90 (T6) dias após o início do tratamento. Na avaliação clínica, observou-se maior redução da secreção ocular, da opacidade e do edema corneano e da vascularização conjuntival. no grupo tacrolimo. No teste de Schirmer, verificou-se produção basal de $6 \pm 4,07$ e 5,86 $\pm 2,85 \mathrm{~mm} / \mathrm{min}$ no TcL e CsA, respectivamente, com aumento significativo da produção lacrimal em ambos os grupos, contudo houve aumento significativo da produção lacrimal a partir dos 15 dias de tratamento no grupo TcL $(17,88 \pm 5,51 \mathrm{~mm} / \mathrm{min})$, mas apenas a partir dos 45 dias no grupo CsA $(11,86 \pm 4,74 \mathrm{~mm} / \mathrm{min})$. Conclui-se que o uso do colírio tacrolimo aumentou em 68,83\% a produção lacrimal em 90 dias de tratamento, comparado com a ciclosporina $(56,82 \%)$, além de diminuir as manifestações clínicas inerentes à CCS, quando comparado à terapia com ciclosporina.

Palavras-chave: cão, ceratoconjuntivite seca, ciclosporina A, tacrolimo

\begin{abstract}
The objective of this study was to evaluate the effects of tacrolimus and cyclosporine on the lacrimal production of dogs with ketaroconjunctivitis sicca (KCS) for 90 days. Tacrolimus $0.02 \%$ (TcL) and $0.1 \%$ cyclosporine (CsA) eye drops were used in 14 dogs with KCS. The animals were randomly assigned to two groups and evaluated before treatment (T0) and at 15 (T1), 30 (T2), 45 (T3), 60 (T4), 75 (T5) and 90 (T6) days after initiation of treatment. Clinical evaluation showed significant reduction of ocular secretion, corneal opacity and edema and conjunctival vascularization in the tacrolimus group. Schirmer test showed basal lacrimal production of $6 \pm 4.07$ and $5.86 \pm 2.85 \mathrm{~mm} / \mathrm{min}$ for TcL and CsA, respectively, with significant increase in lacrimal production in both groups. There was a significant increase in lacrimal production after 15 days of treatment in the TcL group $(17.88 \pm 5.51 \mathrm{~mm} / \mathrm{min})$, but only after 45 days in the CsA group (11.86 $\pm 4.74 \mathrm{~mm} / \mathrm{min})$. Tacrolimus drops increased lacrimal production in $68.83 \%$ after 90 days of treatment, compared to cyclosporine (56.82\%), and also reduced clinical manifestations related to KCS when compared to cyclosporine.
\end{abstract}

Keywords: dog, ketaroconjunctivitis sicca, cyclosporine A, tacrolimus

Recebido em 28 de fevereiro de 2017

Aceito em 25 de agosto de 2017

*Autor para correspondência (corresponding author)

E-mail: fa.bono@gmail.com 


\section{INTRODUÇÃO}

A ceratoconjuntivite seca (CCS) é uma desordem multifatorial do filme lacrimal, caracterizada por lesões no epitélio corneano, inflamação da superfície ocular e manifestações clínicas de desconforto devido à instabilidade lacrimal (Kim et al., 2009; Lin e Yiu, 2014; Daull et al., 2016).

À medida que a patogênese da doença avança, há perda substancial de produção da lágrima em razão de linfócitos infiltrantes, secreção de citocinas inflamatórias, eventual destruição das glândulas lacrimais, disfunção da glândula lacrimal remanescente e diminuição do reflexo lacrimal em resposta aos impulsos nervosos sensoriais (Stern et al., 2004).

A terapia do olho seco requer abordagem múltipla, incluindo conservação e substituição da lágrima por meio do método clínico (colírio antiinflamatório, lacrimoestimulantes e lacrimomiméticos) e, em alguns casos, cirurgia, com oclusão do ducto lacrimal, transplante de glândula salivar ou transposição do ducto parotídeo (Grahn e Storey, 2004; Siqueira Mendonça et al., 2004; Angélico et al., 2011; Bhavsar, et al., 2011). Diversas terapias estão descritas na literatura, mas os trabalhos mais recentes apontam resultados promissores dos imunomoduladores ciclosporina e tacrolimo como terapia adjuvante no tratamento da CCS. Tanto a ciclosporina (CsA) quanto o tacrolimo atuam na redução da infiltração linfocitária nas glândulas lacrimais e na supressão das células epiteliais mediante modulação da atividade das células $\mathrm{T}$ por meio da inibição da enzima calcineurina (Gao et al., 1998; Sloper et al., 1999; Kihara et al., 2009; Abud et al., 2016).

Diante disso, o presente estudo objetiva avaliar os efeitos do colírio de tacrolimo $0,02 \%$ e de ciclosporina $0,1 \%$ na produção lacrimal de cães com CCS, durante 90 dias.

\section{MATERIAL E MÉTODOS}

O estudo foi desenvolvido no Hospital Veterinário da Universidade Federal do Paraná Setor Palotina, previamente aprovado pelo Comitê de Ética no Uso de Animais do Setor Palotina da UFPR, Protocolo $n^{\circ}$ 02/2016. Todos os tutores assinaram termo de consentimento livre e esclarecido para participação do estudo, prontificando-se a aplicar os medicamentos e a retornar para as reavaliações.

Participaram do presente estudo 14 cães de diferentes raças, pesos e idades, com diagnóstico de CCS, caracterizado por baixa produção lacrimal, confirmado pelo teste lacrimal de Schirmer menor que $10 \mathrm{~mm} /$ minuto. Cães com anormalidades anatômicas (entrópio, triquíase, distiquíase, ectrópio) e que apresentassem doenças sistêmicas em curso (por exemplo, cinomose) eram excluídos do estudo. Foi considerado como unidade amostral cada olho afetado, totalizando 15 olhos com diagnóstico de CCS.

Os cães foram distribuídos aleatoriamente em dois grupos experimentais: grupo TcL, que recebeu colírio tacrolimo $0,02 \%$ (DrogaVet, Brasil), a cada 12 horas, e grupo CsA, que recebeu colírio ciclosporina A 0,1\% (DrogaVet, Brasil), a cada 12 horas. Além dos fármacos em teste, todos os animais foram medicados com colírio dextrana 70-hipromelose (duasorb*) (Lacrima Plus ${ }^{\circledR}$ Alcon Laboratório, Brasil), a cada quatro horas, e com colírio tobramicina 0,3\% (Tobrex $^{\circledR}$, Alcon Laboratório, Brasil), a cada seis horas, durante todo o período experimental.

Os cães foram avaliados antes do início do tratamento (T0) e aos 15 (T1), 30 (T2), 45 (T3), 60 (T4), 75 (T5) e 90 (T6) dias após o início do tratamento. Em cada avaliação, foi realizado um teste lacrimal de Schirmer (TLS), acompanhado por dois avaliadores que desconheciam $\mathrm{o}$ tratamento a que o olho estava sendo submetido. A produção de lágrimas foi medida utilizando-se, uma tira de Schirmer padronizada (DrogaVet $\left.{ }^{\circledR}\right)$, colocada no fórnice conjuntival lateral ou medial inferior antes da manipulação do olho ou da administração de medicação tópica. As pálpebras foram suavemente mantidas fechadas durante a duração do teste de um minuto. Como as tiras de Schirmer podem variar em sua capacidade de absorção entre os fabricantes, todas as tiras usadas eram do mesmo fabricante e lote. Adicionalmente, cada animal foi submetido à avaliação clínica oftalmológica antes do início do tratamento (T0) e ao final do tratamento (T6), sendo observada presença de secreção ocular, presença de opacidade e edema corneano e hiperemia conjuntival. O teste de Schirmer e a 
avaliação clínica eram realizados em ambos os olhos de todos os cães.

Avaliou-se a eficácia no aumento de produção lacrimal de cada grupo e a comparação entre os protocolos conforme o tempo de administração estabelecido (quinzenal), por meio de análise de variância (ANOVA) e comparação pelos testes Dunett e $t$ não pareado, com nível de significância de $5 \%$. As alterações clínicas foram avaliadas de forma descritiva.

\section{RESULTADOS E DISCUSSÃO}

Dos 14 cães inicialmente selecionados, dois foram desclassificados durante o estudo, um devido ao desconforto após aplicação do colírio de CsA e outro pelo desacordo do proprietário com as datas de retorno. A produção lacrimal basal (média \pm desvio-padrão) era de $6 \pm 4,07$ e $5,86 \pm 2,85 \mathrm{~mm} / \mathrm{min}$ para $\mathrm{TcL}$ e CsA, respectivamente.

No teste de Schirmer, observou-se aumento significativo da produção lacrimal em ambos os grupos $(68,83$ e $56,82 \%$ para $\mathrm{TcL}$ e CsA, respectivamente). $\mathrm{Na}$ comparação entre tempos em cada grupo, houve aumento significativo da produção lacrimal a partir dos 15 dias de tratamento do grupo TcL $(17,88 \pm 5,51 \mathrm{~mm} / \mathrm{min}) \mathrm{e}$ a partir dos 45 dias no grupo CsA $(9,29 \pm 8,32 \mathrm{~mm} / \mathrm{min}$ ) (Fig. 1). Kihara et al. (2009) defendem que o tacrolimo tem efeito imunossupressor até 100 vezes superior comparado à CsA. É possível que essa maior potência seja responsável pela precocidade da resposta ao tratamento observada no presente estudo. Embora os pré-fármacos ciclosporina e tacrolimo se liguem a diferentes moléculas-alvo, ambos os fármacos inibem a ativação das células $\mathrm{T}$ da mesma forma (Stepkowski, 2000). Lauema et al. (1997), em seu estudo in vitro, relatam que a permeabilidade do tacrolimo foi maior comparada à CsA, o que seria uma hipótese da eficácia superior do tacrolimo.

Adicionalmente, na comparação dois a dois entre grupos nos diferentes tempos, observou-se diferença significativa entre os grupos aos 15 dias de tratamento, com maior produção lacrimal no grupo tacrolimo, o que condiz com Williams (2008), que relata o efeito lacrimomimético do tacrolimo superior ao da CsA. Da mesma forma,
Berdoulay et al. (2005), ao estudarem o efeito do tacrolimo em cães responsivos e não responsivos à CsA, observaram aumento maior que $5 \mathrm{~mm}$ no TLS em $26,7 \%$ e em $51,1 \%$ dos olhos nos grupos responsivos e não responsivos à CsA, respectivamente. Contudo, apesar dos melhores resultados relacionados ao tacrolimo, Best et al. (2014) afirmam que o tacrolimo é um fármaco indicado apenas quando a CsA não apresentar efeito desejado. Segundo esses autores, a resposta ao tratamento com CsA é observada apenas após 90 dias de aplicação contínua, com intervalo de 12 horas, mas, ainda assim, recomendam-no como primeira escolha no tratamento da CCS. No presente estudo, os animais do grupo CsA demonstraram melhora apenas aos 45 dias de tratamento, confirmando que a CsA tem efeito mais tardio quando comparada ao TcL, no qual se observou melhora aos 15 dias, e, mesmo após o final do tratamento, a produção lacrimal foi superior no grupo TcL. Resultado semelhante foi observado por Radziejewski e Balicki (2016), que mostraram que tacrolimo apresentou diferença significativa no primeiro mês de tratamento e a CsA apenas no segundo mês, mesmo utilizando uma dose superior de CsA $(0,75 \%)$ que no presente estudo.

Com relação à avaliação clínica, em T0 observou-se opacidade e edema de córnea em $50 \%$ dos animais do grupo tacrolimo e em $28,5 \%$ nos animais do grupo CsA. Ao final do tratamento, houve redução dessa alteração em $75 \%$ dos cães acometidos no grupo TcL e nenhuma redução nos cães do grupo CsA, diferentemente do resultado de Radziejewski e Balicki (2016), que observaram aumento da transparência ocular em $74 \%$ no grupo tacrolimo e em $54,8 \%$ no grupo CsA.

A presença de secreção ocular em T0 foi observada em $75 \%$ dos cães do grupo TcL e em $57,1 \%$ dos cães do grupo CsA. Ao final do período experimental, observaram-se $100 \%$ de melhora no grupo TcL e apenas $50 \%$ no grupo CsA. Apesar de Herrera et al. (2007) terem observado melhora em $100 \%$ dos animais tratados com ciclosporina, essa redução foi discreta. No presente estudo, a avaliação da presença de secreção foi dicotômica (presente ou ausente), portanto é possível que um maior número de animais do grupo CsA tenha apresentado uma resposta discreta, como descrito por Herrera et al. (2007). Radziejewski e Balicki 
(2016) também observaram diminuição de secreção em $78 \%$ dos animais do grupo tacrolimo (0,02\%/BID) e em $89 \%$ dos animais do grupo ciclosporina $(0,75 \% /$ TID $)$, mesmo com avaliação por menor tempo (60 dias) e maior

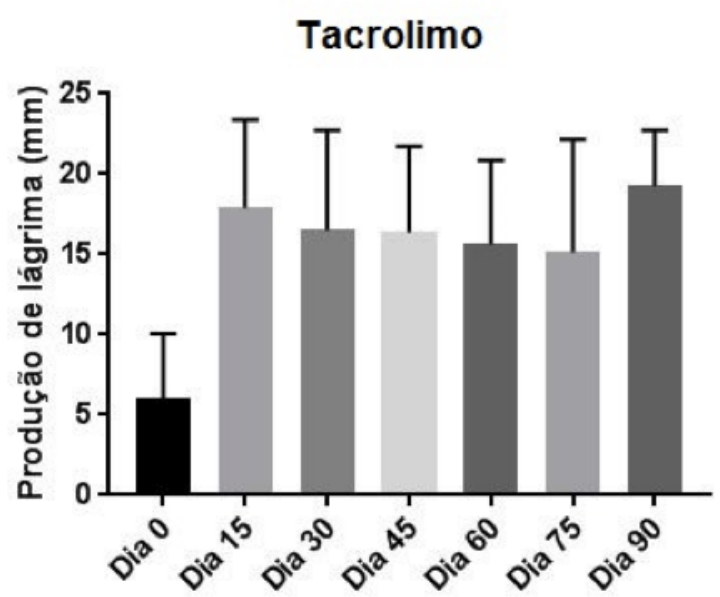

concentração e frequência da ciclosporina do que a do presente estudo.

A hiperemia conjuntival foi observada em $100 \%$ dos cães em ambos os grupos em T0, porém houve redução em $87,5 \%$ dos cães no grupo tacrolimo e em 57,14\% no grupo CsA.

\section{Ciclosporina}

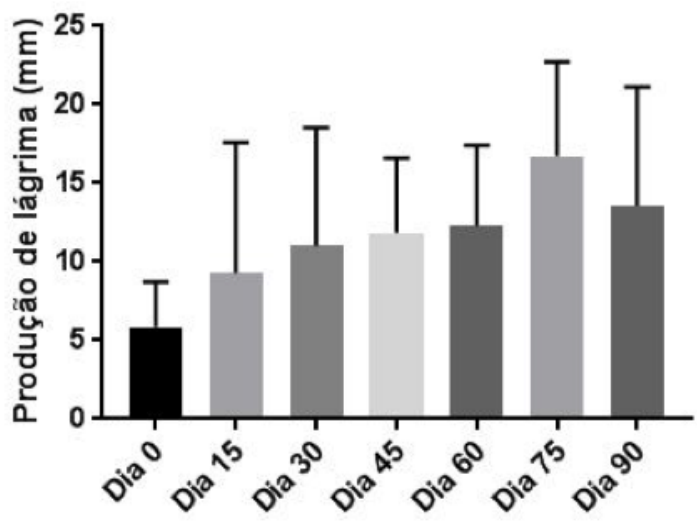

Figura 1. Produção de lágrima $(\mathrm{mm} / \mathrm{min})$ pelo teste lacrimal de Schirmer em cães com ceratoconjuntivite seca, tratados com tacrolimo e ciclosporina durante 90 dias.

A inflamação se desenvolve na superfície ocular à medida que a produção lacrimal diminui (Stern et al., 1998), desencadeando os sinais observados em T0, em ambos os grupos, que incluíram hiperemia conjuntival, secreção ocular mucoide, vascularização e opacidade corneana. Tais sinais também foram descritos por Herrera et al. (2007), Sanchez et al. (2007) e Pigatto et al. (2008). Pigatto et al. (2008) citam que, em casos mais crônicos, pode haver também pigmentação corneana, como visto em um dos cães do grupo TcL.

À medida que a inflamação diminui e a integridade epitelial da superfície ocular melhora, as terminações nervosas sensoriais na córnea e na conjuntiva podem ser estimuladas, de forma mais eficaz, ao piscar e com outros estímulos, e os sinais neurais para a glândula lacrimal normalizam, o que melhora a quantidade e a qualidade da lágrima (Sall et al., 2000). A melhora da inflamação acontece pela ação da ciclosporina ou do tacrolimo, que inibem a atividade da calcineurina e, consequentemente, a desfosforilação do fator nuclear da proteína das células $\mathrm{T}$ ativada, um fator de transcrição necessário para a expressão de citocinas inflamatórias (Tocci et al., 1989; Schreibe e Crabtree, 1992), que diminui os sinais inflamatórios e exerce sua atividade imunossupressiva.

\section{CONCLUSÕES}

No que tange aos aspectos inflamação, produção lacrimal e evolução das manifestações clínicas, verificou-se diferença clínica e estatística em relação à eficiência do colírio TcL $0,02 \%$ quando comparado ao colírio CsA $0,1 \%$, sendo o tacrolimo superior à ciclosporina.

\section{AGRADECIMENTOS}

A empresa DrogaVet, pela doação dos materiais necessários para a execução do experimento; às ONGs Arca de Noé e ASFA, que auxiliaram na seleção dos animais, a todos os envolvidos do Hospital Veterinário da UFPR - Setor Palotina. 


\section{REFERÊNCIAS}

ABUD, T.B.; AMPARO, F.; SABOO, U.S. et al. A clinical trial comparing the safety and efficacy of topical tacrolimus versus methylprednisolone in ocular graft-versus-host disease. Ophthalmology, v.123, p.1449-1457, 2016.

ANGÉLICO, G.T.; RANZANI, J.J.T.; BRANDÃO, C.V.S. et al. Transplante de glândulas salivares menores no tratamento da ceratoconjuntivite seca em cães. Arq. Bras. Med. Vet. Zootec., v.63, p.1087-1092, 2011.

BERDOULAY, A.; ENGLISH, R.V.; NADELSTEIN, B. Effect of topical $0.02 \%$ tacrolimus aqueous suspension on tear production in dogs with keratoconjunctivitis sicca. Vet. Ophthalmol., v.8, p.225-232, 2005 .

BEST, L.J.; HENDRIX, D.V.; WARD, D.A. Diagnosis \& treatment of keratoconjunctivitis sicca in dogs. Today's Vet. Pract., v.4, p.16-22, 2014.

BHAVSAR, A.S.; SAMIR, G.B.; SUNITA, M.J. A review on recent advances in dry eye: pathogenesis and management. Oman J. Ophthalmol., v.4, p.50-56, 2011.

DAULL, P.; FERAILLE, L.; BARABINO, S. et al. Efficacy of a new topical cationic emulsion of cyclosporine a on dry eye clinical signs in an experimental mouse model of dry eye. Exp. Eye Res., v.26, p.159-164, 2016.

GAO, J.; SCHWALB, T.A.; ADDEO, J.V. et al. The role of apoptosis in the pathogenesis of canine keratoconjunctivitis sicca: the effect of topical Cyclosporin A therapy. Cornea, v.17, p.654-663, 1998.

GRAHN, B.H.; STOREY, E.S. Lacrimostimulants and lacrimomimetics. Vet. Clin. Small Anim., v.34, p.739753, 2004.

HERRERA, H.D.; WEICHSLER, N.; GÓMEZ, J.R.; JALÓN, J.A.G. Severe, unilateral, unresponsive keratoconjunctivitis sicca in 16 juvenile Yorkshire Terriers. Vet. Ophthalmol., v.10, p.285-288, 2007.

KIHARA, Y.; MATSUNO, N.; MIJITI, A. et al. Comparative study of the cellular pharmacodynamics of calcineurin inhibitors between patients with chronic renal failure awaiting renal transplantation and cirrhosis patients awaiting liver transplantation. Cell. Transplant., v.18, p.639-646, 2009.

KIM, E.C.; CHOI, J.S.; JOO, C.K. A comparison of vitamin a and cyclosporine a $0.05 \%$ eye drops for treatment of dry eye syndrome. Am. J. Ophthalmol., v.147, p.206-213, 2009.
LAUEMA, A.I.; SURBER, C.; MAIBACH, H.I. Absorption of topical tacrolimus (FK506) in vitro through human skin: comparison with cyclosporine A. Skin Pharmacol. Physiol., v.10, p.230-234, 1997.

LIN, H.; YIU, S.C. Dry eye disease: a review of diagnostic approaches and treatments. Saudi $J$. Ophthalmol., v.28, p.173-181, 2014.

PIGATTO, J.A.T.; PEREIRA, F.Q.; ALMEIDA, A.C.V.R. et al. Ceratoconjuntivite seca em cãesrevisão de literatura. Vet. Foco, v.5, p.191-200, 2008.

RADZIEJEWSKI, K.; BALICKI, I. Comparative clinical evaluation of tacrolimus and cyclosporine eye drops for the treatment of canine keratoconjunctivitis sicca. Acta Vet. Hung., v.64, p.313-329, 2016.

SALL, K.; STEVENSON, O.D.; MUNDORF, T.K.; REIS, B.L. Two multicenter, randomized studies of the efficacy and safety of cyclosporine ophthalmic emulsion in moderate to severe dry eye disease. Ophthalmology, v.107, p.631-639, 2000.

SANCHEZ, R.F.; INNOCENT, G.; MOULD, J.; BILLSON, F.M. Canine keratoconjunctivitis sicca: disease trends in a review of 229 cases. J. Small. Anim. Pract., v.48, p.211-217, 2007.

SCHREIBER, S.L.; CRABTREE, G.R. The mechanism of action of cyclosporin A and FK506. Immunol. Today, v.13, p.136-142, 1992.

SIQUEIRA MENDONÇA, C.; CARNEIRO, F.O.; CUNHA, G.N.; COELHO, H.E. Topografia da papila parotidea em cães sem raça definida. Biotemas, v.17, p.217-224, 2004.

SLOPER, C.M.L.; POWELL, R.J.; DUA, H.S. Tacrolimus (FK506) in the treatment of posterior uveitis refractory to cyclosporine. Ophthalmology, v.106, p.723-728, 1999.

STEPKOWSKI, S.M. Molecular targets for existing and novel immunosuppressive drugs. Expet. Rev. Mol. Med., v.2, p.1-23, 2000.

STERN, M.E.; BEUERMAN, R.W.; FOX, R.I. et al. The pathology of dry eye: the interaction between the ocular surface and lacrimal glands. Cornea, v.17, p.584-589, 1998.

STERN, M.E.; GAO, J.; SIEMASKO, K.F. et al. The role of the lacrimal functional unit in the pathophysiology of dry eye. Exp. Eye Res., v.78, p.409-416, 2004.

TOCCI, M.J.; MATKOVICH, D.A.; COLLIER, K.A. et al. The immunosuppressant FK506 selectively inhibits expression of early $\mathrm{T}$ cell activation genes. $J$. Immunol., v.143, p.718-726, 1989.

WILLIAMS, D.L. Immunopathogenesis of keratoconjunctivitis sicca in the dog. Vet. Clin. Small Anim., v.38, p.251-268. 2008. 